\title{
Global alliances and independent domination in some classes of graphs
}

\author{
Odile Favaron \\ LRI, UMR 8623, Univ Paris-Sud F-91405 Orsay, France; \\ CNRS, F-91405 Orsay \\ of@lri.fr
}

Submitted: Nov 21, 2007; Accepted: Sep 22, 2008; Published: Sep 29, 2008

Mathematics Subject Classification: $05 \mathrm{C} 69$

\begin{abstract}
A dominating set $S$ of a graph $G$ is a global (strong) defensive alliance if for every vertex $v \in S$, the number of neighbors $v$ has in $S$ plus one is at least (greater than) the number of neighbors it has in $V \backslash S$. The dominating set $S$ is a global (strong) offensive alliance if for every vertex $v \in V \backslash S$, the number of neighbors $v$ has in $S$ is at least (greater than) the number of neighbors it has in $V \backslash S$ plus one. The minimum cardinality of a global defensive (strong defensive, offensive, strong offensive) alliance is denoted by $\gamma_{a}(G)\left(\gamma_{\hat{a}}(G), \gamma_{o}(G), \gamma_{\hat{o}}(G)\right)$.

We compare each of the four parameters $\gamma_{a}, \gamma_{\hat{a}}, \gamma_{o}, \gamma_{\hat{o}}$ to the independent domination number $i$. We show that

$i(G) \leq \gamma_{a}^{2}(G)-\gamma_{a}(G)+1$ and $i(G) \leq \gamma_{\hat{a}}^{2}(G)-2 \gamma_{\hat{a}}(G)+2$ for every graph

$i(G) \leq \gamma_{a}^{2}(G) / 4+\gamma_{a}(G)$ and $i(G) \leq \gamma_{\hat{a}}^{2}(G) / 4+\gamma_{\hat{a}}(G) / 2$ for every bipartite graph

$i(G) \leq 2 \gamma_{a}(G)-1$ and $i(G)=3 \gamma_{\hat{a}}(G) / 2-1$ for every tree

and describe the extremal graphs,

and that $\gamma_{o}(T) \leq 2 i(T)-1$ and $i(T) \leq \gamma_{\hat{o}}(T)-1$ for every tree.

We use a lemma stating that $\beta(T)+2 i(T) \geq n+1$ in every tree $T$ of order $n$ and independence number $\beta(T)$.

Keywords: independence, domination, alliance, bipartite graph, tree.
\end{abstract}

\section{Introduction}

We consider simple graphs $G=(V(G), E(G))$ with vertex set $V(G)$, edge set $E(G)$, order $n(G)=|V(G)|$ and size $m(G)=|E(G)|(V, E, n, m$ when no ambiguity is possible). The degree in $G$ of a vertex $v$ is denoted by $d_{G}(v)$, or simply $d(v)$, and the number of neighbors of $v$ in a subset $S$ of $V$ by $d_{S}(v)$.

A subset $S$ of vertices is dominating if every vertex of $V \backslash S$ has at least one neighbor in $S$, and independent if no two vertices of $S$ are adjacent. It is well known that a dominating 
set is independent if and only if it is a maximal independent set and that in every graph, $\gamma(G) \leq i(G) \leq \beta(G)$ where $\gamma(G)$ and $i(G)$ are respectively the minimum cardinality of a dominating set and of an independent dominating set and $\beta(G)$ is the maximum cardinality of an independent set. Alliances are defined in [6] as follows. A subset $S \subseteq V$ is a defensive alliance (respectively strong defensive alliance) if $d_{V \backslash S}(v) \leq d_{S}(v)+1$ (respectively $d_{V \backslash S}(v)<d_{S}(v)+1$ ) for every $v \in S$. In other words, every vertex of $S$ together with its neighbors in $S$ is as strong as (respectively stronger than) the coalition of its neighbors out of $S$. The subset $S$ is an offensive alliance (respectively a strong offensive alliance) if $d_{S}(v) \geq d_{V \backslash S}(v)+1$ (respectively $d_{S}(v)>d_{V \backslash S}(v)+1$ ) for every vertex $v \in V \backslash S$ dominated by $S$. In other words, every vertex out of $S$ and dominated by $S$ together with its neighbors out of $S$ is not stronger (respectively weaker) than the coalition of its neighbors in $S$. Alliances of any sort are global if they dominate $G$. The minimal cardinality of a global defensive (respectively strong defensive, offensive, strong offensive) alliance of $G$ is denoted by $\gamma_{a}(G)$ (respectively $\gamma_{\hat{a}}(G), \gamma_{o}(G), \gamma_{\hat{o}}(G)$ ). Clearly $\gamma(G) \leq \gamma_{a}(G) \leq \gamma_{\hat{a}}(G)$ and $\gamma(G) \leq \gamma_{o}(G) \leq \gamma_{\hat{o}}(G)$ for every graph $G$. Similar notions exist under the name of coalitions or monopolies. In particular a monopoly is a global defensive and offensive alliance [7].

Properties of global alliances can be found in several papers, some of them are referenced below $[1,2,3,4,5,8,9]$, in particular relationships between alliance parameters and other graph parameters valid for all graphs or in some classes of graphs. In [3], Chellali and Haynes compared in trees the independence number $\beta$ to the four parameters $\gamma_{a}, \gamma_{\hat{a}}, \gamma_{o}, \gamma_{\hat{o}}$ by establishing some inequalities between them. They also noticed that for trees $T$, the independence domination number $i$ is "incomparable" to some global alliance parameters in that sense that $i(T)$ can be smaller than $\gamma_{a}(T)$ or $\gamma_{o}(T)$, or greater than $\gamma_{\hat{a}}(T)$. Our purpose is to replace in the comparisons $\beta$ by $i$ and to refine the notion of incomparability by asking for instance if $i(G)$, even when greater than $\gamma_{a}(G)$, cannot be bounded by a function of $\gamma_{a}(G)$. Moreover, we do not limit ourselves to trees.

The principe of the study is to determine for each value of $\mu$ among $\gamma_{a}, \gamma_{\hat{a}}, \gamma_{o}, \gamma_{\hat{o}}$ and for a class $\mathcal{C}$ of graphs whether a function $f$ such that $i(G) \leq f(\mu(G))$ or $\mu(G) \leq f(i(G))$ for every $G$ in $\mathcal{C}$ can exist, and when the answer is positive, to determine such a function. We consider the classes of all graphs, bipartite graphs and trees. Each of the following four sections is devoted to the comparison of $i(G)$ with one of the four alliance parameters.

We give first some more precisions on the notation. The neighborhood $N(v)$ of a vertex is the set of vertices adjacent with it and the closed neighborhood is $N[v]=N(v) \cup\{v\}$. If $A \subseteq V, N_{A}(v)=N(v) \cap A$. The subgraph induced by $A$ in $G$ is denoted by $G[A]$ and its size by $m(A)$. The graph $G-A$ is obtained from $G$ by deleting the vertices of $A$ and the edges incident with them. If $F$ a subset of edges of $G$, then $G-F$ is the graph obtained by deleting all the edges of $F$ from $G$. In several places we consider a graph $G$ constructed from a graph $S$ by adding some new vertices and edges. To lighten the writing, we often use in this case the notation $|S|$ for $n(S)$ or $|V(S)|$. The corona of a graph is obtained by attaching a pendant edge at each vertex of $G$. 


\section{Global defensive alliances}

For the star $G$ of order $n, i(G)=1, \gamma_{a}(G)=\left\lceil\frac{n}{2}\right\rceil$ and $\gamma_{\hat{a}}(G)=\left\lceil\frac{n+1}{2}\right\rceil$. Therefore no general bound of the type $\gamma_{a}(G) \leq f(i(G))$ or $\gamma_{\hat{a}}(G) \leq g(i(G))$ can be satisfied by every graph, even if we reduce ourselves to the class of trees.

We study now the existence of a function $f$ such that $i(G) \leq f\left(\gamma_{a}(G)\right)$ for every general graph, bipartite graph or tree.

\section{Definitions 1}

(1) $\mathcal{F}_{1}$ is the family of graphs obtained from a clique $S \sim K_{k}$ by attaching $k=d_{S}(u)+1$ leaves at each vertex $u$ of $S$.

(2) $\mathcal{F}_{2}$ is the family of bipartite graphs obtained from a balanced complete bipartite graph $S \sim K_{k, k}$ by attaching $k+1=d_{S}(u)+1$ leaves at each vertex $u$ of $S$.

(3) $\mathcal{F}_{3}$ is the family of trees obtained from a tree $S$ by attaching a set $L_{u}$ of $d_{S}(u)+1$ leaves at each vertex $u$ of $S$.

Proposition 1 (1) If $G \in \mathcal{F}_{1}$ then $i(G)=\gamma_{a}^{2}(G)-\gamma_{a}(G)+1$.

(2) If $G \in \mathcal{F}_{2}$ then $i(G)=\gamma_{a}^{2}(G) / 4+\gamma_{a}(G)$.

(3) If $G \in \mathcal{F}_{3}$ then $i(G)=2 \gamma_{a}(G)-1$.

Proof: If $G \in \mathcal{F}_{i}$ with $1 \leq i \leq 3$, then $V(S)$ is a minimum dominating set and a defensive alliance of $G$. Therefore $\gamma(G) \leq \gamma_{a}(G) \leq|S|=\gamma(G)$ and thus $\gamma_{a}(G)=|S|$.

(1) If $G \in \mathcal{F}_{1}$, i.e., $S \sim K_{k}$, then $i(G)=1+(k-1) k=|S|^{2}-|S|+1$.

(2) If $G \in \mathcal{F}_{2}$, i.e., $S \sim K_{k, k}$, then $|S|=2 k$ and $i(G)=k+k(k+1)=|S|^{2} / 4+|S|$.

(3) Let $T \in \mathcal{F}_{3}$ be constructed from a tree $S$ with bipartition classes $X$ and $Y$. Every maximal independent set $I$ of $T$ can be written as $I=(I \cap V(S)) \cup\left(\cup_{u \in V(S) \backslash I} L(u)\right)$. Therefore

$$
|I|=|I \cap V(S)|+\sum_{u \in V(S) \backslash I}\left(d_{S}(u)+1\right)=|V(S)|+\sum_{u \in V(S) \backslash I} d_{S}(u) .
$$

In the sum $\sum_{u \in V(S) \backslash I} d_{S}(u)$, the edges of $S$ between $V(S) \backslash I$ and $I$ are counted once and the $m(S-I)$ edges joining two vertices in $V(S) \backslash I$ are counted twice. Hence

$$
\begin{gathered}
\sum_{u \in V(S) \backslash I} d_{S}(u)=m(S)+m(S-I) \geq m(S), \quad \text { and } \\
|I| \geq|V(S)|+m(S)=2 n(S)-1 .
\end{gathered}
$$

For the particular sets $I=X \cup\left(\cup_{u \in Y} L(u)\right)$ and $I=Y \cup\left(\cup_{u \in X} L(u)\right), m(V(S) \backslash I)=\emptyset$ and $|I|=2 n(S)-1$. Therefore, $i(T)=2 n(S)-1=2 \gamma_{a}(T)-1$.

Theorem $1(1)$ Every graph $G$ satisfies $i(G) \leq \gamma_{a}^{2}(G)-\gamma_{a}(G)+1$ with equality if and only if $G \in \mathcal{F}_{1}$.

(2) Every bipartite graph $G$ satisfies $i(G) \leq \gamma_{a}^{2}(G) / 4+\gamma_{a}(G)$ with equality if and only if $G \in \mathcal{F}_{2}$. 
(3) Every tree $G$ satisfies $i(G) \leq 2 \gamma_{a}(G)-1$ with equality if and only if $G \in \mathcal{F}_{3}$.

Proof Let $S$ be a $\gamma_{a}(G)$-set, $W$ a maximal independent set of $G[S]$, and $B$ a maximal independent set of $G\left[N_{V \backslash S}(S) \backslash N_{V \backslash S}(W)\right]$. Then $W \cup B$ is a maximal independent set of $G$ and $i(G) \leq|W|+|B|$. For each $v \in S$, let $L(v)=N_{V \backslash S}(v)$. Since $S$ is a defensive alliance, $|L(v)| \leq d_{S}(v)+1$ for every $v \in S$, and since the defensive alliance is dominating,

$$
\begin{aligned}
|B| & \leq\left|N_{V \backslash S}(S \backslash W)\right| \leq \sum_{v \in S \backslash W}|L(v)| \leq \sum_{v \in S \backslash W}\left(d_{S}(v)+1\right) \\
& \leq|S|-|W|+\sum_{v \in S \backslash W} d_{S}(v) .
\end{aligned}
$$

Therefore

$$
i(G) \leq|S|+\sum_{v \in S \backslash W} d_{S}(v) .
$$

(1) In every graph, $d_{S}(v) \leq|S|-1$. Therefore $i(G) \leq|S|+(|S|-|W|)(|S|-1)$ with $|W| \geq 1$. Hence

$$
i(G) \leq|S|^{2}-|S|+1=\gamma_{a}^{2}(G)-\gamma_{a}(G)+1 .
$$

If $i(G)=|S|^{2}-|S|+1$ then $|W|=1$ and $d_{S}(v)=|S|-1$ for every $v \in S \backslash W$, i. e., $S$ is a clique and $W$ consists of any vertex $w$ of $S$. Moreover, for any $w \in S$, equality in (1) gives $|B|=\left|N_{V \backslash S}(S \backslash\{w\})\right|$, i. e., $\mid N_{V \backslash S}(S \backslash\{w\})$ is independent, and $\left|N_{V \backslash S}(S \backslash\{w\})\right|=\sum_{S \backslash\{w\}}|L(v)|=\sum_{S \backslash\{w\}}\left(d_{S}(v)+1\right)$, i. e., all the sets $L(v)$ for $v \in S$ are disjoint, independent and of order $d_{S}(v)+1$. Therefore $G \in \mathcal{F}_{1}$. The converse is true by Proposition 1(1).

(2) Suppose now $G$ bipartite. Let $U$ be the set of isolated vertices of $G[S]$ and $X \cup Y$ a bipartition of $G[S \backslash U]$. If we take $W=X \cup U$ then we get by (2),

$$
i(G) \leq|S|+\sum_{v \in Y} d_{S}(v)=|S|+m(S) .
$$

Since $G[S]$ is bipartite, $m(S) \leq|S|^{2} / 4$ and thus

$$
i(G) \leq|S|^{2} / 4+|S|=\gamma_{a}^{2}(G) / 4+\gamma_{a}(G) .
$$

If $i(G)=|S|^{2} / 4+|S|$, then $m(S)=|S|^{2} / 4$, i.e., $U=\emptyset$ and $G[S]$ is a complete balanced bipartite graph. Moreover, equality in (1) implies that all the sets $L(v)$ for $v \in Y$ are disjoint and of respective orders $d_{S}(v)+1$. By symmetry between $X$ and $Y$, the same property holds for all $v \in X$. Hence $G \in \mathcal{F}_{2}$. The converse is true by Proposition $1(2)$.

(3) If the bipartite graph $G$ is a tree, then $G[S]$ is a forest. By $(3), i(G) \leq|S|+m(S)$ with $m(S) \leq|S|-1$. Therefore

$$
i(G) \leq 2|S|-1=2 \gamma_{a}(S)-1
$$

If $i(G)=2|S|-1$, then $m(S)=|S|-1$, i. e., $G[S]$ is a tree, the sets $L(v)$ are all disjoint for $v \in Y$ and of respective order $d_{S}(v)+1$, and the same holds for all $v \in X$ by symmetry between $X$ and $Y$. Therefore $G \in \mathcal{F}_{3}$. The converse is true by Proposition 1(3). 


\section{Global strong defensive alliances}

As shown by the example of stars in the previous section, we have only to look for bounds on the type $i(G) \leq g\left(\gamma_{\hat{a}}(G)\right)$ valid for every graph, bipartite graph or tree. Since $\gamma_{a}(G) \leq \gamma_{\hat{a}}(G)$ for every graph, the increasing functions $f$ such that $i(G) \leq f\left(\gamma_{a}(G)\right)$ which were defined inTheorem 1 are convenient but possibly too large. We are looking for sharp bounds.

\section{Definitions 2}

(1) $\mathcal{G}_{1}$ is the family of graphs obtained from a clique $S \sim K_{k}$ by attaching $k-1=d_{S}(u)$ leaves at each vertex $u$ of $S$.

$(2) \mathcal{G}_{2}$ is the family of bipartite graphs obtained from a complete balanced bipartite graph $S \sim K_{k, k}$ by attaching $k=d_{S}(u)$ leaves at each vertex $u$ of $S$.

(3) $\mathcal{S}$ is the family of trees $S$ such that for every maximal independent set $J$ of $S$, the number of components of the forest $S-J$ is at most $|S| / 2$.

$\mathcal{G}_{3}$ is the family of trees obtained from a tree $S$ of $\mathcal{S}$ by attaching a set $L(u)$ of $d_{S}(u)$ leaves at each vertex $u$ of $S$.

Observation Every tree $S$ in $\mathcal{S}$ is balanced since if $X$ and $Y$ are the two classes of the bipartition of $S$ with $|X| \leq|Y|$, then $S-X$ has $|Y|$ components. Every tree $T$ in $\mathcal{G}_{3}$ constructed from $S \in \mathcal{S}$ is balanced of order $|T|=|S|+\sum_{u \in V(S)} d_{S}(u)=|S|+2 m(S)=$ $3|S|-2$.

Lemma 1 Let $T$ be a tree constructed from a balanced tree $S$ by attaching a set $L(u)$ of $d_{S}(u)$ leaves at each vertex $u$ of $S$. Let $I$ be a maximal independent set of $T$ and $q$ the number of components of the forest induced in $T$ by $V(S) \backslash I$. Then $|I|=2|S|-q-1$.

Proof Every maximal independent set of $T$ has the form $I=(V(S) \cap I) \cup\left(\cup_{u \in V(S) \backslash I} L(u)\right)$. Hence $|I|=|I \cap V(S)|+\sum_{u \in V(S) \backslash I} d_{S}(u)$. As in the proof of Proposition 1(3), $\sum_{u \in V(S) \backslash I} d_{S}(u)$ $=m(S)+m(S-I)$ and thus $|I|=|I \cap V(S)|+m(S)+m(S-I)$. Since $S$ is a tree and $S-I$ a forest with $q$ components, $m(S)=|S|-1$ and $m(S-I)=|V(S) \backslash I|-q$. Therefore $|I|=|I \cap V(S)|+(|S|-1)+(|S|-|I \cap V(S)|-q)=2|S|-q-1$.

Proposition 2 (1) Every graph $G$ of $\mathcal{G}_{1}$ satisfies $i(G)=\gamma_{\hat{a}}^{2}(G)-2 \gamma_{\hat{a}}(G)+2$.

(2) Every graph $G$ of $\mathcal{G}_{2}$ satisfies $i(G)=\gamma_{\hat{a}}^{2}(G) / 4+\gamma_{\hat{a}}(G) / 2$.

(3) Every tree $G$ of $\mathcal{G}_{3}$ satisfies $i(G)=3 \gamma_{\hat{a}}(G) / 2-1$.

Proof If $G$ is a graph of $\mathcal{G}_{i}, 1 \leq i \leq 3$, constructed from a graph $S$ by attaching $d_{S}(u)$ leaves at each vertex $u$ of $S$, then $V(S)$ is a global strong defensive alliance and a minimum dominating set of $G$. Therefore $\gamma(G) \leq \gamma_{\hat{a}}(G) \leq|S|=\gamma(G)$ and thus $\gamma_{\hat{a}}(G)=|S|$.

(1) If $S$ is a clique $K_{k}$, then $\gamma_{\hat{a}}(G)=k$ and $i(G)=(k-1)^{2}+1=\gamma_{\hat{a}}^{2}(G)-2 \gamma_{\hat{a}}(G)+2$. 
(2) If $S$ is a complete balanced bipartite graph $K_{k, k}$, then $\gamma_{\hat{a}}(G)=2 k$ and $i(G)=$ $k(k+1)=\gamma_{\hat{a}}^{2}(G) / 4+\gamma_{\hat{a}}(G) / 2$.

(3) Let $S$ be a tree of $\mathcal{S}$ of bipartition $X \cup Y$ with $|X|=|Y|$ and let $I=(V(S) \cap$ $I) \cup\left(\cup_{u \in V(S) \backslash I} L(u)\right.$ be a $i(G)$-set such that $|I \cap V(S)|$ is maximum. By Lemma $1,|I|=$ $2|S|-q-1$ where $q$ is the number of components of the forest induced by $V(S) \backslash I$. If the independent set $I \cap V(S)$ is not maximal in $S$, let $u$ be a vertex of $S$ not dominated by $I \cap V(S)$. Then $I$ contains the set $L(u)$ and the maximal independent set $(I \backslash L(u)) \cup\{u\}$ of $G$ is smaller than $I$ if $|L(u)| \geq 2$ or contradicts the choice of $I$ if $|L(u)|=1$. Therefore $I \cap V(S)$ is a maximal independent set $J$ of $S$. Since $S \in \mathcal{S}, q \leq|S| / 2$. Therefore $i(G)=|I| \geq 3|S| / 2-1$. Now the set $X \cup_{y \in Y} L(u)$ is a maximal independent set of $G$ of order $|G| / 2=3|S| / 2-1$. Hence $i(G)=3|S| / 2-1=3 \gamma_{\hat{a}}(G) / 2-1$.

Theorem 2 (1) Every graph $G$ satisfies $i(G) \leq \gamma_{\hat{a}}^{2}(G)-2 \gamma_{\hat{a}}(G)+2$ with equality if and only if $G \in \mathcal{G}_{1}$.

(2) Every bipartite graph $G$ without isolated vertices satisfies $i(G) \leq \gamma_{\hat{a}}^{2}(G) / 4+\gamma_{\hat{a}}(G) / 2$ with equality if and only if $G \in \mathcal{G}_{2}$.

(3) Every tree $G$ of order $n \geq 2$ satisfies $i(G)=3 \gamma_{\hat{a}}(G) / 2-1$ with equality if and only if $G \in \mathcal{G}_{3}$.

Proof We follow the same idea as in the proof of Theorem 1. Let $G$ be a graph, $S$ a $\gamma_{\hat{a}}(G)$-set, $W$ a maximal independent set of $G$ and $B$ a maximal independent set of $N_{V \backslash S}(S) \backslash N_{V \backslash S}(W)$. Then $W \cup B$ is a maximal independent set of $G$ and $i(G) \leq|W|+|B|$. Moreover since $S$ is a strong defensive alliance, the set $L(v)=N_{V \backslash S}(v)$ has order at most $d_{S}(v)$ for every vertex $v$ in $S$. Therefore

$$
|B| \leq\left|N_{V \backslash S}(S \backslash W)\right| \leq \sum_{v \in S \backslash W}|L(v)| \leq \sum_{S \backslash W} d_{S}(v)
$$

and

$$
i(G) \leq|W|+\sum_{v \in S \backslash W} d_{S}(v) .
$$

(1) In every graph, $d_{S}(v) \leq|S|-1$. Hence by (5),

$$
i(G) \leq|W|+(|S|-|W|)(|S|-1)=|S|(|S|-1)-|W|(|S|-2) \text { with }|W| \geq 1 .
$$

Therefore

$$
i(G) \leq|S|^{2}-2|S|+2=\gamma_{\hat{a}}^{2}(G)-2 \gamma_{\hat{a}}(G)+2 .
$$

If $i(G)=\gamma_{\hat{a}}^{2}(G)-2 \gamma_{\hat{a}}(G)+2$, then $|W|=1$ and $d_{S}(v)=|S|-1$ for every $v \in S$, i. e., $S$ is a clique and $W$ consists of any unique vertex $w$ of $S$. Moreover equality everywhere in (4) shows that all the sets $L(v)$ for $v \in S$ are independent and disjoint. Therefore $G \in \mathcal{G}_{1}$. The converse is true by Proposition 2(1).

(2) Suppose now $G$ bipartite without isolated vertices. Since $S$ is a strong defensive alliance, $G[S]$ has no isolated vertices. Consider the unique bipartition $X_{i} \cup Y_{i}$ of each 
component $S_{i}$ of $G[S], 1 \leq i \leq p$, with $\left|X_{i}\right| \leq\left|Y_{i}\right|$ and let $X=\cup_{1 \leq i \leq p} X_{i}, Y=\cup_{1 \leq i \leq p} Y_{i}$. Then $|X| \leq|S| / 2 \leq|Y|$. By taking $W=X$, we get by (5)

$$
i(G) \leq|X|+\sum_{v \in Y} d_{S}(v) \leq|S| / 2+m(S) .
$$

Since $G[S]$ is bipartite, $m(S) \leq|S|^{2} / 4$. Therefore

$$
i(G) \leq|S|^{2} / 4+|S| / 2=\gamma_{\hat{a}}^{2}(G) / 4+\gamma_{\hat{a}}(G) / 2 .
$$

If $i(G)=\gamma_{\hat{a}}^{2}(G) / 4+\gamma_{\hat{a}}(G) / 2$, then $|X|=|S| / 2$ and $m(S)=|S|^{2} / 4$, i. e., $G[S]$ is a complete balanced bipartite graph. Moreover by equality in (4), the sets $L(v)$ have respective order $d_{S}(v)$ and are all disjoint. By symmetry between $X$ and $Y$, the same property holds for all $v \in X$. Therefore $G \in \mathcal{G}_{2}$. The converse is true by Proposition 2(2).

(3) If the bipartite graph $G$ is a tree, then $G[S]$ is a forest and $m(S) \leq|S|-1$. By (6),

$$
i(G) \leq 3|S| / 2-1=3 \gamma_{\hat{a}}(G) / 2-1 .
$$

If $i(G)=3 \gamma_{\hat{a}}(G) / 2-1$, then $|X|=|S| / 2$ and $m(S)=|S|-1$, i. e., $G[S]$ is a balanced tree. Moreover the sets $L(v)$ have respective orders $d_{S}(v)$ and are all disjoint. Let $J$ be any maximal independent set of $G[S]$ and $q$ the number of components of the forest induced by $S \backslash J$. The set $I=J \bigcup_{v \in S \backslash J} L(v)$ is a maximal independent set of $G$. By Lemma 1, $|I|=2|S|-q-1$. Therefore $3|S| / 2-1=i(G) \leq 2|S|-q-1$. Hence $q \leq|S| / 2, G[S] \in \mathcal{S}$ and $G \in \mathcal{G}_{3}$. The converse is true by Proposition 2(3).

\section{Global offensive alliances}

The double star $T$ obtained by adding an edge between the centers of two stars $K_{1, p}$ satisfes $i(T)=1+n / 2$ and $\gamma_{o}(T)=2$. Therefore no general bound of the type $i(G) \leq f\left(\gamma_{o}(G)\right)$ can exist, even if we limit ourselves to the class of trees.

We are now interested in the existence of bounds of the type $\gamma_{o}(G) \leq f(i(G))$. The bipartite graph $G$ obtained by deleting one edge from a complete bipartite graph $K_{p, p}$ satisfies $i(G)=2$ and $\gamma_{o}(G)=n / 2$. Therefore no general bound $\gamma_{o}(G) \leq f(i(G))$ can exist, even in the class of bipartite graphs. To study the possibility of such a bound valid for all trees, we first give a result relating $\beta(G)$ and $i(G)$ in this class.

Lemma 2 For every tree $T$ of order $n, \beta(T)+2 i(T) \geq n+1$ and the bound is sharp.

Proof Let $T=(V, E)$ be a tree of order $n \geq 2, I$ a $i(T)$-set and $F$ the set of edges of $T[V \backslash I]$. Then $T-F$ is a forest with $q \leq i(T)$ components and since $T$ is a tree, $|F|=q-1 \leq i(T)-1$. Let $A$ be a set of vertices of $V \backslash I$ containing at least one extremity of each edge in $F$ and such that $|A| \leq|F|$. Each vertex of $V \backslash(A \cup I)$ has all its neighbors in $A \cup I$. Hence $V \backslash(A \cup I)$ is an independent set of order $n-(|I|+|A|) \geq$ 
$n-(|I|+|F|) \geq n-(2 i(T)-1)$. Therefore $\beta(T)+2 i(T) \geq n+1$. The result is clearly true for $n=1$.

The star $T \sim K_{1, n-1}$ satisfies $\beta(T)+2 i(T)=n+1$. More generally, let $T$ be the trees obtained from paths $P_{3 k+1}=u_{1} u_{2} \cdots u_{3 k+1}$ by attaching at each vertex $u_{3 i+1}, 0 \leq i \leq k$, a non-empty set $L_{i}$ of new leaves. For these trees, $I=\left\{u_{1}, u_{4}, \cdots, u_{3 k+1}\right\}$ is a $i(T)$-set and $B=\left(\cup_{0 \leq i \leq k} L_{i}\right) \cup\left\{u_{2}, u_{5}, \cdots, u_{3 k-1}\right\}$ is a $\beta(T)$-set of order $n-|I|-\left|\left\{u_{3}, u_{6}, \cdots, u_{3 k}\right\}\right|=$ $n-|I|-k$. Hence $i(T)=k+1, \beta(T)=n-2 k-1$ and $\beta(T)+2 i(T)=n+1$.

Theorem 3 For every tree $T, \gamma_{o}(T) \leq 2 i(T)-1$ and the bound is sharp.

Proof As already observed in [3], for every independent set of a connected graph $G$ of order $n \geq 2$, the set $V \backslash S$ is a global offensive alliance of $G$. Hence $\gamma_{o}(G) \leq n-\beta(G)$. If the graph is a tree $T$ then, by Lemma $2, \gamma_{o}(T) \leq 2 i(T)-1$ and this result remains clearly true for $n=1$. For the trees satisfying $\beta(T)+2 i(T)=n+1$ which are described above, $I \cup\left\{u_{3}, u_{6}, \cdots, u_{3 k}\right\}$ is a $\gamma_{o}(G)$-set. Therefore they also satisfy $\gamma_{o}(T)=2 i(T)-1$.

Remark The inequality $\gamma_{o}(G) \leq n-\beta(G)$ in the proof of Theorem 3 shows that $\gamma_{o}(G) \leq$ $\beta(G)$ for every graph without isolates such that $\beta(G) \geq n / 2$, and in particular for bipartite graphs. This property was proved in [3] for trees.

\section{$5 \quad$ Global strong offensive alliances}

Since all the leaves of any graph $G$ belong to every $\gamma_{\hat{o}}(G)$-set, every star $T$ satisfies $\gamma_{\hat{o}}(T)=n-1$ while $i(T)=1$. Therefore no general bound $\gamma_{\hat{o}}(G) \leq f(i(G))$ can exist, even in the class of trees.

We are now interested in the existence of bounds of the type $i(G) \leq f\left(\gamma_{\hat{o}}(G)\right)$. The bipartite graph $G$ constructed from a cycle $C_{4}=x y z t x$ by adding an independent set $\left\{u_{1}, \cdots, u_{p}, v_{1}, \cdots, v_{p}\right\}$ of $2 p \geq 4$ vertices and the edges $u_{i} x, u_{i} z, v_{i} y, v_{i} t$ for $1 \leq i \leq p$ satisfies $n=2 p+4, i(G)=n / 2$ and $\gamma_{\hat{o}}(G)=4$. Therefore no general bound $i(G) \leq$ $f\left(\gamma_{\hat{o}}(G)\right)$ can exist, even in the class of bipartite graphs. The following theorem establishes such a bound in the class of trees.

Theorem 4 For every tree $T$ of order $n \geq 2, i(T) \leq \gamma_{\hat{o}}(T)-1$ and the bound is sharp.

Proof It is proved in [3] that every tree satisfies $\beta(T) \leq \gamma_{\hat{o}}(T)$. Hence $i(T) \leq \gamma_{\hat{o}}(T)$. We prove that the equality is impossible. If $i(T)=\gamma_{\hat{o}}(T)$ then $i(T)=\beta(T)$ and $T$ is a well-covered tree. Therefore $\beta(T)=n / 2$ and $T$ is the corona of a tree of vertex set $W$. Let $A$ be a $\gamma_{\hat{o}}(T)$-set. Then $A$ contains the set $L$ of leaves of $T$ and a dominating set of $W$ since every vertex of $V(T) \backslash A$ must have at least two neighbors in $A$. Therefore $|A| \geq 1+n / 2$ which contradicts $\beta(T)=\gamma_{\hat{o}}(T)$. Hence $i(T) \leq \gamma_{\hat{o}}(T)-1$.

Equality occurs if $i(T)=\beta(T)=\gamma_{\hat{o}}(T)-1$, or if $i(T)=\beta(T)-1$ and $\beta(T)=\gamma_{\hat{o}}(T)$. The coronas of stars, for which $\gamma(W)=1$, are the only trees satisfying the first equalities. The subdivided stars, obtained by subdividing once each edge of a star, are examples of graphs satisfying the second ones. 


\section{References}

[1] A. Cami, H. Balakrishnan, N. Deo and R. D. Dutton, On the complexity of finding optimal global alliances, J. Combin. Math. Combin. Comput. 58 (2006), 23-31.

[2] M. Chellali, Offensive alliances in bipartite graphs, submitted.

[3] M. Chellali and T. W. Haynes, Global alliances and independence in trees, Discuss. Math. Graph theory 27 (2007), no 1, 19-27.

[4] T. W. Haynes, S. T. Hedetniemi amd M. A. Henning, Global defensive alliances in graphs, The Electronic Journal of Combinatorics 10 (2003), no R47.

[5] T. W. Haynes, S. T. Hedetniemi amd M. A. Henning, A characterization of trees with equal domination and global strong alliance numbers, Utilitas Math. 66 (2004), 33-45.

[6] P. Kristiansen, S. A. Hedetniemi and S. T. Hedetniemi, Alliances in graphs, JCMCC 48 (2004), 157-177.

[7] N. Linial, D. Peleg, Y. Rabinovich and M. Saks, Sphere packing and local majorities in graphs, in: Proceedings of the second ISTCS, IEEE Computer Society Press, Silver Spring, MD, 1993, 141-149.

[8] J. A. Rodríguez-Velázquez and J. M. Sigarreta, Global offensive alliances in graphs, Electron. Notes Discrete Math. 25 (2006), 157-164.

[9] J. A. Rodríguez-Velázquez and J. M. Sigarreta, Global alliances in planar graphs, AKCE Int. J. Graphs Comb. 4 (2007), no 1, 83-98. 